\title{
Significance of Occlusion in the Etiology and Treatment of Early, Moderate and Advanced Periodontitis
}

\author{
Sigurd P. Ramfjord* and Major M. Ash, Jr. ${ }^{*}$
}

Concepts describing occlusion as a factor in the initiation, progress, and treatment of periodontal disease have a long and varied history. ${ }^{1-8}$ Although the proposed interactions between occlusal stress and periodontitis have usually involved the lesion of trauma from occlusion, occlusal relationships which interfere with plaque elimination, and/or injure the surface of the gingiva also have been postulated as etiologic factors. Thus malocclusion, irregularity of teeth, crowding, tipping, nonfunction, and faulty marginal ridges have been considered important in clinical periodontics. The present assessment of the role of occlusion in the etiology and treatment of periodontitis will draw upon a number of investigations and epidemological surveys of possible relationships between occlusion and periodontal disease.

\section{SIGNIFICANCE OF TRAUMA FROM OCCLUSION TO PERIODONTAL DISEASE}

Injury to the periodontal tissues as a result of occlusal forces has been defined as the lesion of trauma from occlusion. ${ }^{31,61}$ Furthermore, any excessive force which results in sufficient displacement of a tooth also can produce a traumatic lesion with similar histologic features. However, it is not customary to describe such forces (viz. orthodontic forces) as occlusal forces or to speak of the lesions as being a response to trauma from occlusion.

Histologic characteristics of the lesions of trauma from occlusion have been well documented. ${ }^{29}, 55,60,73,75,78,79$ In only a few instances has the apical movement of the junctional epithelium been related to trauma from occlusion; ${ }^{8,} 47,71,78$ in most well controlled studies, pocket formation has not developed with trauma from occlusion. ${ }^{11,} 61,{ }^{74}$ An unverified explanation for failure of trauma from occlusion to initiate periodontal pockets is the presence of the supracrestal fibers which act as a barrier to the downgrowth of the junctional epithelium. ${ }^{55}$, ${ }^{11}$ Bone changes in the periodontium as a result of trauma from occlusion without an existing inflammation are reversible ${ }^{34,56,52}$ when the forces are discontinued.

When considering trauma from occlusion and peri-

* Professor of Dentistry, University of Michigan, Ann Arbor, MI 48109 . odontitis in a co-destructive relationship ${ }^{26,28,30,59}$ investigators ${ }^{41,51}$ using a monkey model-system were not able to demonstrate a loss of connective tissue attachment. However, they did find that jiggling trauma caused some loss of height of crestal bone and a loss of the overall volume of alveolar bone. ${ }^{41,51}$ Other investigators using a beagle dog model ${ }^{47}$ reported a deepening of experimentally induced periodontal pockets and an acceleration of bone resorption by progressive traumatic occlusion. A number of reasons for the different results of the two model systems have been speculated upon.

The spread of gingival inflammation into the periodontal membrane in association with trauma from occlusion has not been found consistently in animals, in human biopsy or in autopsy material. ${ }^{32,} 42,69$ Although the biological reactions appear to be similar between humans and experimental animals, there may be significant differences between animals as well as between animals and man with respect to the role of trauma from occlusion in the pathogenesis of periodontal disease.

On the basis of evidence from experiments on animals, trauma from occlusion does not initiate gingivitis or periodontitis or accelerate the change of gingivitis to periodontitis. Whether or not trauma from occlusion will accelerate the progress of pocket formation or significantly influence the attachment level is still controversial.

\section{Tooth Mobility}

Increased tooth mobility, with or without bone loss, is often used as the only clinical indicator for trauma from occlusion. However, hypermobility does not necessarily indicate trauma from occlusion, ${ }^{79}$ it may be due to bone loss. ${ }^{53}$ In the absence of additional signs and symptoms, it is a mistake to equate tooth hypermobility with trauma from occlusion. The diagnosis of trauma from occlusion is difficult if not impossible to make on the basis of a single examination inasmuch as the diagnosis requires that ongoing progressive injury be demonstrated. Movement of teeth, repair and compensatory change will often eliminate the occlusal factors producing the trauma and also eliminate the clinical evidence. Thus the trauma from occlusion may be self-limiting. A clinical diagnosis of trauma from occlusion requires evidence of active injury.

Both physiologic adaptation to function and patholog- 
ical response to excessive function are seen clinically as increased tooth mobility, and to separate the two requires more information than tooth hypermobility. The diagnosis is made on the basis of continued or increasing hypermobility, persistent discomfort or tenderness, and radiographic evidence of bone and/or root resorption. Hypermobility in the absence of bone loss due to periodontal disease may be a helpful finding but increased mobility may not relate directly to the severity of periodontal disease. $^{52}$

The presence in radiographs of a widened periodontal space does not necessarily indicate hypermobility due to trauma from occlusion. It may mean that a process of physiologic adaptation is present or that trauma from occlusion has occurred in the past, but has become selflimiting, or that trauma is present at the time. Additional historical evidence of past tenderness or subsequent progressive changes may be of value in making the diagnosis of ongoing injury to the supporting structures.

Hypermobility of individual teeth may persist after completion of periodontal treatment, including occlusal adjustment. The nature of such mobility and what should be done about it, if anything, is a controversial issue. Even so, there has been considerably reduced emphasis on splinting in the last decade. Splinting and occlusal adjustment will be discussed later.

Hypermobility of individual teeth or groups of teeth usually does not increase as a consequence of periodontal treatment alone, but may decrease or remain the same. ${ }^{46 \text {, }}$ ${ }^{64,65}$ Also in the case of a reduced, but healthy periodontium after periodontal therapy, increased mobility does not usually lead to further loss of periodontal support. ${ }^{45}$ However, it is difficult to dismiss the clinical observation that the degree of hypermobility of some teeth (viz. bicuspids) is more significant under certain conditions (viz. abutment for a clasped partial denture) than it would be for other teeth with the same degree of hypermobility but under different conditions. The presence of traumatic rocking forces resulting in progressive injury and increasing mobility may disturb masticatory function and cause discomfort for the patient. Such hypermobility is not acceptable.

Increased mobility may be self-limiting, i.e., reach a point of no increase in mobility. Such is often the case in the mandibular incisor region where a nontraumatic balance between forces and periodontal adaptation develops and function and comfort are not a problem.

Trauma from occlusion has been listed as a possible cause of gingival recession; ${ }^{14,}, 25,33,70,80$ however, recession appears to be more related to plaque than to tooth hypermobility ${ }^{5,6}$ or malocclusion. ${ }^{76}$

\section{Orthodontics, Bruxism and Restorative Dentistry}

Although orthodontic treatment may lead to root resorption without apparent reason, root resorption is more likely to occur with the use of heavy forces, ${ }^{64}$ and is of more significance where periodontal support has already been lost. This does not suggest that age itself is a significant deterrent; orthodontic treatment can be carried out successfully at any age with controlled orthodontic procedures. Orthodontic tilting of teeth in dogs has shown that the periodontal response with loss of attachment can occur with plaque-infected teeth. ${ }^{16,}{ }^{17}$ The transient trauma associated with orthodontics is not a significant etiologic factor with adequate periodontal care.

Both primary and secondary trauma from occlusion may be caused by bruxism. The periodontal significance of bruxism increases with a decrease in periodontal support. Although bruxism tends to lead to compensatory hypertrophy, the presence of bruxism must be considered in the prognosis and treatment of periodontitis.

Faulty dental procedures and restorations may be a significant iatrogenic source of trauma from occlusion. For example, marginal ridge relationships and plunger cusps have been considered to be etiologic factors in periodontal disease. ${ }^{44,12}$ Although clinical observations have supported the importance of marginal ridge relations, ${ }^{38,} 24,58,9$ controlled research on the correlation between pocket depth and marginal ridge discrepancy has not supported these observations. A recent study ${ }^{43}$ found that marginal ridge relations in general are far less important than other local factors such as plaque. Unequal marginal ridge height may not be directly related to food impaction or to periodontal destruction.

The periodontal destruction caused by continuous trauma to abutment teeth can be due to faulty design of partial dentures. ${ }^{60}$ In the presence of faulty types of frame work, clasps and tissue base support, extraction forces on the teeth and gingival irritation may cause an accelerated loss of the abutment teeth.

Habits such as biting on pencils or foreign objects can result in localized destruction of supporting structures. ${ }^{67}$

\section{Trauma from Occlusion and Pathogenesis of Periodontal Disease}

The role of trauma from occlusion in the pathogenesis of periodontal disease is not fully understond, but some provisional conclusions can be drawn from the evidence available at this time. (1) Trauma from occlusion does not initiate or aggravate marginal gingivitis or initiate periodontal pockets; (2) it can cause increased tooth mobility; (3) active trauma tends to accelerate bone loss and pocket formation depending on the presence of local irritants and inflammation; (4) trauma may be perpetuated by bruxism; and (5) it plays a minor role, if any, in the pathogenesis of early to moderate periodontitis.

\section{SIGNIFICANCE OF TOOTH POSITION}

The relationship of occlusion to periodontal disease involves not only trauma from occlusion but also the potential effect of tooth position and irregularity and crowding of teeth on periodontal disease. 
There does not appear to be any significant correlation between malocclusion in terms of a classification (viz. Angle ${ }^{3}$ ) and periodontal disease.$^{20}$ However, when some features of malocclusion are considered separately, the evidence for a positive relationship is conflicting. ${ }^{7,} 13,19$, $36,48,50,63,70$ The potential of direct trauma to the gingiva associated with irregularly placed teeth along with a predisposition to plaque accumulation has led to the concept that such teeth are more likely to develop periodontal disease than teeth in good alignment. Except for cases of direct trauma to the gingival tissues, orthodontic treatment to reduce the likelihood of periodontal disease in later life ${ }^{10}$ has little support. ${ }^{35}$

Several investigators have found a positive relationship between crowding or malalignment of the teeth and the amount of plaque present, ${ }^{2,7,57}$ and between crowding and loss of attachment. ${ }^{1}$ Another investigator reported that the gingival pockets of overlapping teeth were deeper than those of normally positioned teeth. ${ }^{72}$ However, other investigators found that malalignment of the teeth had little or no relationship to gingivitis or periodontitis. ${ }^{4,21,39}$ The determining factor for the severity of gingivitis, whether teeth are crowded or not, is the amount of plaque present. Thus malposed teeth are not a significant periodontal problem in persons with good oral hygiene. ${ }^{1,2}$ It has been suggested that excellent hygiene can just as well be found in the patient with the worst malocclusion as with the best occlusion. ${ }^{68}$

When there is marked protrusion of maxillary anterior teeth, the lip seal may be obstructed which can lead to mouthbreathing and predispose to gingivitis. ${ }^{15}$ The most significant factor is not the crowding of the maxillary anterior teeth but the presence of mouth breathing. ${ }^{40}$

It has been suggested that drifted teeth or those with abnormal proximal inclinations are related to poor oral hygiene and thus such positions are detrimental to periodontal health. ${ }^{19}, 23,27,37,49,62,66$ One study $^{22}$ found a consistent correlation between periodontal destruction and mesially inclined molars, but gingival inflammation was not influenced by either abnormal tooth inclination or tooth drift. Also it was found that mesial inclination predisposes to a slightly increased incidence of tooth mobility. Another investigation ${ }^{4}$ showed no correlation between the severity of periodontal disease in young patients and tooth irregularities on the basis of changes in axial inclination, displacement, and rotation of teeth. Progressive tipping associated with continuous trauma from occlusion can accelerate periodontal breakdown. ${ }^{59}$

\section{Tooth Position and Pathogenesis of Periodontal Disease}

The role of crowding, rotation, tilting, and other specific features of malocclusion in the pathogenesis of periodontal disease has not been studied extensively where objective methods of evaluating plaque have been utilized. Although data from longitudinal human studies are not available, current evidence indicates: (1) there is no significant direct relationship between malocclusion (on the basis of Angle's classification) and the severity of periodontal disease but malocclusion may indirectly affect periodontal health when it is severe enough to interfere with plaque removal; (2) malocclusion, as with impinging overbite, can cause trauma to the gingiva; and (3) severely malposed individual teeth may affect periodontal health.

\section{OCCLUSAL THERAPY AS A PART OF PERIODONTAL TREATMENT}

Since some aspects of the relationship between occlusion and the etiology of periodontitis are not entirely clear, there is no agreement on requisites for occlusal treatment in periodontics. One reason for the lack of agreement arises from the remaining controversy about the importance of trauma from occlusion as an etiologic factor in periodontitis. Another problem is the lack of established criteria for identifying periodontal trauma from occlusion. An additional difficulty is the paucity of longitudinal data on the beneficial effects of the various forms of occlusal therapy. Despite these problems and disagreements, however, current concepts concerning trauma from occlusion do provide guidance for the role of occlusal therapy in the treatment of periodontal disease.

\section{General Principles}

A general principle for the initial treatment of early, moderate and advanced periodontitis in which the etiologic factors are both bacterial plaque and occlusal factors is to eliminate and control first the plaque and then the occlusal factors, except where delay may unfavorably influence later treatment, or cause discomfort to the patient. In such instances simultaneous, palliative treatment may be indicated.

A general principle for the control of occlusal factors is to establish a stable occlusion with the least interference to plaque control and periodontal maintenance.

A general principle for treating persistent tooth hypermobility following periodontal treatment and occlusal adjustment is to maintain determined oral hygiene and professional periodontal care, and to splint only when such mobility interferes with the health and comfort of the patient and/or is progressively increasing.

Periodontal trauma from occlusion represents a lesion in the periodontium originating in dysfunctional occlusal relations which have to be eliminated to restore periodontal health. A periodontist or another dentist who completely ignores occlusal therapy under those circumstances will fail to attain complete periodontal health for the patient, although he may succeed in eliminating gingival inflammation.

Occlusal therapy may also help to reduce periodontal pockets for tipped teeth and restore functional capacity to teeth with progressive mobility and trauma from occlusion. Obviously occlusal therapy, in itself, cannot 
cure gingivitis or periodontitis caused by bacterial activity, but it may facilitate plaque control and management of patients with periodontal disease.

The following modalities of occlusal therapy may be applicable as part of periodontal treatment: (1) Orthodontic treatment. (2) Temporary splinting. (3) Bite planes. (4) Occlusal adjustment. (5) Restorative dentistry. (6) Splinting, long term.

\section{Orthodontic Treatment}

Since there is no significant correlation between malocclusion (Angle's classification) and severity of periodontal disease, orthodontic treatment of malocclusion should not be considered a routine preventive or therapeutic procedure in periodontics. However, there are a number of more specific aspects of combined malocclusion and periodontal disease in which orthodontic treatment may augment or even be essential to optimal results of periodontal therapy.

a. Impinging overbite or shearing occlusion with trauma to gingival tissues usually requires orthodontic treatment, or in extreme cases a combination of orthodontic and surgical procedures, often augmented by occlusal adjustment and restorative dentistry.

b. Lack of lip seal and mouth breather's gingivitis may indicate the need for orthodontic treatment.

c. Functional anterior crossbite often is accompanied by an unstable jiggling type of occlusion which seems to aggravate periodontitis; in addition, attaining functional comfort often will require orthodontic treatment.

d. Uprighting tipped teeth may reduce periodontal pocket depth and will facilitate restorative dentistry and plaque control.

e. Correction of severe malposition of teeth in relation to the alveolar process is needed to secure a long term favorable prognosis.

f. Malposition of teeth in gingival fibromatosis often requires orthodontic treatment for optimal esthetic and functional results.

g. Extensive open bite with contacts only on the last molars often has associated periodontal problems and orthodontic treatment may be beneficial.

h. Extruded anterior teeth with advanced periodontal disease and mobility may be positioned orthodontically into the alveolar process and subsequently treated by reattachment procedures. Forced eruption of teeth with intrabony pockets may also reduce the pocket depth.

Orthodontic treatment should always be done after complete scaling and instruction in plaque control, but prior to periodontal surgery or restorative procedures.

\section{Temporary Splinting}

The purpose of a temporary splint is to reduce occlusal forces and stabilize teeth for a limited period of time.
The alleged value of temporary splinting with regard to promotion of healing and regeneration of periodontal structures has not been substantiated by research findings. Mobility of teeth may be decreased by reduction of inflammation, both with and without splinting, and the value of temporary splinting in periodontal treatment has not been established beyond facilitating scaling and occlusal adjustment.

A recent investigation ${ }^{18}$ has reported that the response 1 year after periodontal treatment was more favorable with regard to gain of attachment in teeth having little or no increase in mobility than in teeth with definite or high increases in mobility. During 7 years of subsequent maintenance care there was no effect on attachment level and pocket depth attributable to higher than normal mobility. It would therefore be interesting to see if temporary splinting prior to periodontal therapy would lead to a more favorable result of the periodontal treatment than in nonsplinted teeth with equal elevation of mobility levels. Such experiments are underway.

Temporary splints using bonding techniques are effective and easy to use. They are also used for temporary replacement of lost teeth until the periodontal treatment is completed. Indications are that temporary splints reduce tooth mobility only as long as they are in place. When such splints are removed, the presplinting degree of mobility will return.

\section{Bite Planes}

Bite-planes have become increasingly popular in many aspects of occlusal therapy. For patients needing periodontal treatment, they are used to eliminate trauma from occlusion, bruxism, and temporomandibular joint or muscle pain, as well as to stabilize mobile teeth and prevent hypereruption of teeth without antagonists. A bite-plane is commonly used following orthodontic repositioning of teeth with advanced bone loss when the patient for some reason cannot have permanent splinting. The best type is the maxillary bite-plane with flat occlusal surface, centric stops for all opposing teeth, and a cuspid rise area for posterior disclusion in lateral and protrusive excursions. This appliance will decrease the occlusal load for single teeth, and also decrease the total muscle activity.

\section{Occlusal Adjustment}

The ultimate value of occlusal adjustment as part of periodontal treatment has not been established in clinical trials. However, periodontal trauma from occlusion represents a lesion which should be eliminated in order to have a healthy masticatory system. Occlusal adjustment is one modality of therapy for elimination of such trauma. An occlusal adjustment does contribute to occlusal stability ${ }^{74}$ Other modalities may also be needed, but if the trauma can be eliminated and the occlusion stabilized by occlusal adjustment, this is usually the method of choice for either primary and secondary 
trauma from occlusion. Occlusal adjustment may also eliminate food impaction and gingival impingement in some instances.

The need for adjustment should be based on a definite diagnosis of the presence of a traumatic lesion rather than the location of some occlusal interferences, which may be of no significance. Only a few patients with early and moderate periodontitis have trauma from occlusion justifying occlusal adjustment. In advanced periodontitis with extensive loss of periodontal support one may encounter increased mobility of the teeth with or without actual evidence of trauma. If there are signs and symptoms of trauma, occlusal adjustment should be considered. However, adjustment is more controversial in cases with increased mobility but without discomfort or roentgenologic evidence of traumatic lesions. For such patients occlusal adjustment also may be recommended since increased mobility signifies at least a compensatory adaptation of the periodontal structures to forces in excess of optimum. The closer the forces can be gotten to an optimal load for the remaining periodontium, the better is the functional result of the periodontal treatment and the smaller are the chances for increasing mobility and trauma with a further decrease in supporting structures and/or increase in occlusal load. This may not eliminate the abnormally increased mobility, but it may, in addition to other forms of periodontal therapy, stabilize the result without permanent splinting.

\section{Restorative Dentistry}

Restorative dentistry aimed at correction of occlusion may be indicated as part of periodontal treatment for patients with food impaction, impinging overbite or various forms of unstable occlusion.

Part of restorative dentistry is "permanent" or longterm splinting of teeth. The purposes of long-term splinting are to eliminate or prevent trauma from occlusion, and to enhance occlusal stability and comfort as well as esthetics. Because of technical and financial problems associated with permanent splinting of teeth, it should be considered only after other forms of occlusal therapy have been ruled out as being inadequate.

Progressive trauma from occlusion following occlusal adjustment and periodontal therapy may be expressed as soreness of the teeth to pressure of percussion during and after function, continuous migration or tipping of teeth, and radiographic evidence of ongoing resorption of the alveolar bone several months after occlusal adjustment. These are all indications for permanent splinting of the teeth, since these findings are diagnostic of traumatic periodontal lesions. Splinting may also be indicated for the maintenance of teeth in the desired position after orthodontic treatment of teeth with advanced bone loss.

\section{CONCLUDING REMARKS}

Elimination of lesions diagnostic of trauma from oc- clusion is an essential part of complete periodontal therapy and restoration of health in the masticatory system for such patients. This can be achieved by orthodontic treatment, temporary splinting, bite-planes, occlusal adjustment and permanent splinting of teeth. Occlusal therapy may be required during periodontal treatment for trauma from occlusion and to enhance occlusal stability at any stage of periodontitis, but is most often needed in advanced periodontitis. Splinting of hypermobility of self-limiting trauma from occlusion is not indicated. Splinting may be required in addition to occlusal adjustment in moderate to severe periodontitis when trauma from occlusion is progressive.

\section{REFERENCES}

1. Ainamo, J.: Relationship between malalignment of teeth and periodontal disease. Scand J Dent Res 80: 104, 1972.

2. Alexander, A. G., and Tipnis, A. K.: The effect of irregularity of teeth and the degree of overbite and overjet on the gingival health. A study of 400 subjects. Br Dent $J$ 128: 539, 1970.

3. Angle, E. H.: Classification of malocclusion. Dent Cosmos 41: 248, 1899.

4. Beagrie, G. S., and James, G. A.: The association of posterior teeth irregularity and periodontal disease. Br Dent J 113: 239, 1962.

5. Bernimoulin, J. P.: Recherches cliniques relatives aux recession gingivales dans la parodontolyse involutive. Schweiz Monatsschr Zahnheilkd 24: 60, 1974.

6. Bernimoulin, J. P., and Curilovic, Z.: Gingival recession and tooth mobility. J Clin Periodont 4: 107, 1977.

7. Bilimoria, K. F.: Malocclusion-its role in the causation of periodontal disease. J All-India Dent Assoc 35: 293, 1963.

8. Box, H. K.: Experimental traumatogenic occlusion in sheep. Oral Health 25: 9, 1935.

9. Burch, J. G.: Periodontal considerations in operative dentistry. $J$ Prosthet Dent 34: 156, 1975.

10. Burke, P.: Orthodontics in theory and practice. $B r$ Dent $J$ 127: $88,1969$.

11. Comar, M. D., Kollar, J. A., and Gargiolo, A. W.: Local irritation and occlusal trauma as co-factors in the periodontal disease process. J Periodontol 40: 193, 1969.

12. Diamond, M.: Dental Anatomy. New York, Macmillan, 1929.

13. Ditto, W. M., and Hall, D. C.: The survey of 143 periodontal cases in terms of age and malocclusion. Am J Orthod 40: 234, 1954.

14. Emslie, R. D.: Localized gingival recession. Int Dent $J$ 8: 18, 1958.

15. Emslie, R.: The incisal relationship and periodontal disease Parodontologie 12: 15, 1958.

16. Ericsson, I., Thilander, B., Lindhe, J., and Okamoto, H.: The effect of orthodontic tilting movements on the periodontal tissues of infected and noninfected dentitions in dogs. J Clin Periodont 4: 278, 1977.

17. Ericsson, I., Thilander, B., and Lindhe, J.: Influence of tipping movements on experimental periodontitis in dogs. $J$ Dent Res 56: (Special Issue A) 144, paper $H$ 407, (Feb.) 1977.

18. Fleszar, T. J., Knowles, J. W., Morrison, E. C., Burgett, F. G., Nissle, R. R., and Ramfjord, S. P.: Tooth mobility and periodontal therapy. J Clin Periodont 7: 495, 1980.

19. Geiger, A. M.: Occlusion in periodontal disease. J Periodontol 36: 378,1965 .

20. Geiger, A. M., Wasserman, B. H., and Thompson, R. H., Jr.: Relationship of occlusion and periodontal disease. V. Relation of classification of occlusion to periodontal status and gingival inflammation. J Periodontol 43: 554, 1972.

21. Geiger, A. M., Wasserman, B. H., and Turgeon, L. R.: Relation of occlusion and periodontal disease. VIII. Relationship of crowding 
and spacing to periodontal destruction and gingival inflammation. $J$ Periodontol 45: 43, 1974.

22. Geiger, A. M., and Wasserman, B. H.: Relationship of occlusion and periodontal disease. Part XI. Relation of axial inclination (mesialdistal) and tooth drift to periodontal disease. $J$ Periodontol 51: 283, 1980.

23. Geiger, A. M.: Occlusal studies in 188 consecutive cases of periodontal disease. Am J Orthod 48: 330, 1962.

24. Glickman, I.: Clinical Periodontology. Philadelphia, W. B. Saunders, 1972.

25. Glickman, I.: Clinical Periodontology. ed 3, p 101. Philadelphia, W. B. Saunders, 1964.

26. Glickman, I.: Inflammation and trauma from occlusion: Codestructive factors in chronic periodontal disease. $J$ Periodontol 34: 5 , 1963.

27. Glickman, I.: Clinical Periodontology. Philadelphia, W. B. Saunders Co. 1953.

28. Glickman, I., and Smulow, J. B.: The combined effects of inflammation and trauma from occlusion in periodontitis. Int Dent $J$ 19: $393,1969$.

29. Glickman, I., and Smulow, J.: Adaptive alterations in the periodontium of the rhesus monkey in chronic trauma from occlusion. $J$ Periodontol 29: 101, 1968.

30. Glickman, I., and Weiss, L.: Role of trauma from occlusion in initiation of periodontal pocket formation in experimental animals. $J$ Periodontol 26: 14, 1955.

31. Glickman, I., and Zander, H. A.: Discussion of role of occlusion in the etiology and treatment of periodontal disease. J Dent Res (Suppl. to 2) 50: $199,1971$.

32. Goldman, H. M.: Extension of exudate into supporting structures of teeth in marginal periodontitis. $J$ Periodontol 28: 175, 1957.

33. Gorman, W. J.: Prevalence and etiology of gingival recession. $J$ Periodontol 38: 316, 1967.

34. Gottlieb, B., and Orban, B.: Die Veranderung der Geivebe bei ubermassiger Beanspruchung der Zahne. Leipzig, Georg Thieme Verlag, 1931

35. Gould, M. S. E., and Picton, D. C. A.: The relation between irregularities of teeth and periodontal disease. Br. Dent J 121: 20, 1966

36. Hellgren, A.: The association between crowding of the teeth and gingivitis. Report of 32nd Congress of European Orthodontic Society, p. 134-140, The Hague, Netherlands, Albani, 1956.

37. Hirschfeld, I.: Individual missing teeth. J Am Dent Assoc 24: 67, 1937.

38. Hirschfeld, I.: Food impaction. J Am Dent Assoc 17: 1504, 1930.

39. Ingervall, B., Jacobsson, U., and Nyman, S.: A clinical study of the relationship between crowding of teeth, plaque and gingival condition. J Clin Periodont 4: 214, 1977.

40. Jacobson, L., and Linder-Aronson, S.: Crowding and gingivitis: A comparison between mouthbreathers and nosebreathers. Scand $J$ Dent Res 80: 500, 1972

41. Kantor, M., Polson, A. M., and Zander, H. A.: Alveolar bone regeneration after removal of inflammatory and traumatic factors. $J$ Periodontol 47: 687, 1976.

42. Kenney, E. G.: A histologic study of incisal dysfunction and gingival inflammation in the rhesus monkey. $J$ Periodontol 42: 3, 1971 .

43. Kepic, T. J., and O'Leary, T. J.: Role of marginal ridge relationships as an etiologic factor in periodontal disease. $J$ Periodontol 49: $570,1978$.

44. Kraus, B. S., Jordan, R. E., and Abrams, L.: Dental Anatomy and Occlusion. Baltimore, The Williams and Wilkins Co, 1969.

45. Lindhe, J., and Ericsson, I.: The influence of trauma from occlusion on reduced but healthy periodontal tissues in dogs. J Clin Periodont 3: 110, 1976.

46. Lindhe, J., and Nyman, S.: The effect of plaque control and surgical pocket elimination on the establishment and maintenance of periodontal health. A longitudinal study of periodontal therapy in cases of advanced disease. $J$ Clin Periodont 2: 67, 1975.

47. Lindhe, J., and Svanberg, G.: Influence of trauma from occlusion on progression of experimental periodontitis in the beagle dog. $J$ Clin Periodont 1: 3, 1974.
48. Massler, M., and Savara, B. S.: Relation of gingivitis to dental caries and malocclusion in children 14-17 years of age. $J$ Periodontol 22: $87,1951$.

49. McCall, J. O.: Tooth malposition as a factor in periodontal disease. $N Y J$ Dent 1: 23, 1931.

50. McCombie, F., and Slothard, $0 .:$ Relationship between gingivitis and other dental conditions. Can Dent Assoc 30: 506, 1964.

51. Meitnev, S. W.: Codestructive factors of marginal periodontitis and repetitive mechanical injury. $J$ Dent Res Special issue C, 54: C78C85, 1975 .

52. Muhlemann, H., and Herzog, H.: Tooth mobility and microscopic tissue changes produced by experimental occlusal trauma. Helv Odontol Acta 5: 33, 1961.

53. Muhlemann, H. R., and Rateitschabe, K. H.: Zur "erhohten Zahnbeweglichkeit. Schweiz Monatsschr Zahnheilkd 75: 85, 1965.

54. O'Leary, T. J., Drake, R. B., Jividen, G. J., and Allen, M. F.: The incidence of recession in young males: Relationship to gingival and plaque scores. Periodontics 6: 109, 1967.

55. Polson, A. M., Meitner, S. W., and Zander, H. A.: Trauma and progression of marginal periodontitis in squirrel monkeys III. Adaptation of interproximal alveolar bone to repetitive injury. $J$ Periodont Res 11: 279, 1976.

56. Polson, A. M., Meitner, S. W., and Zander, H. A.: Trauma and progression of marginal periodontitis in squirrel monkeys. IV Reversibility of bone loss due to trauma alone and trauma superimposed upon periodontitis. J Periodont Res 11: 290, 1976.

57. Poulton, D. R., and Aaronson, S. H.: The relation between occlusion and periodontal status. Am J Orthol 47: 690, 1961.

58. Prichard, J. F.: Advanced Periodontal Disease/Surgical and Prosthetic Management. ed 2. Philadelphia, W. B. Saunders Co, 1972.

59. Ramfjord, S. P., and Ash, M. M., Jr.: Periodontology and Periodontics, Philadelphia, W. B. Saunders, p. 184, 1979.

60. Ramfjord, S. P., and Ash, M. M., Jr.: Periodontology and Periodontics, p 178 Philadelphia, W. B. Saunders Co, 1979.

61. Ramfjord, S. P., Kerr, D. A., and Ash, M. M.: World Workshop in Periodontics, $\mathrm{p} 271$, Ann Arbor, Michigan, The University of Michigan, 1966.

62. Ramfjord, S. P., and Ash, M. M., Jr.: Occlusion. Philadelphia, W. B. Saunders Co, 1971.

63. Razdan, D. P., and Chawla, T. N.: Malocclusion-its association with periodontal disease. J Indian Dent Assoc 42: 39, 1970.

64. Reitan, K.: Biomechanical principles and reactions. Graber, T. M. and Swain, B. F. (eds). Current Orthodontic Concepts and Techniques, ed 2, vol 1. Philadelphia, W. B. Saunders Co, 1975.

65. Rosling, B., Nyman, S., and Lindhe, J.: The effect of systematic plaque control on bone regeneration in infrabony pockets. $J$ Clin Periodont 3: 38, 1976

66. Sippy, B. O.: The effects of loss of the first permanent molar. $J$ Am Dent Assoc 14: 1953, 1927.

67. Sorrin, S.: Habit, an etiologic factor of periodontal disease. Dent Dig 41: 290, 1935.

68. Stahl, S. S.: The need for orthodontic treatment: A periodontist's point of view. Int Dent $J$ 25: 242, 1975.

69. Stahl, S. S.: The response of the periodontium to combined gingival inflammation and occlusofunctional stress in four human surgical specimens. Periodontics 6: 14, 1968.

70. Stillman, P. R.: Early clinical evidence of disease in the gingiva and periocementum. $J$ Dent Res 3: 25, 1921

71. Stones, H. H.: An experimental investigation into the association of traumatic occlusion with periodontal disease. Proc $R$ Soc Med 31: $479,1938$.

72. Suomi, J. D.: Periodontal disease and oral hygiene in an institutionalized population: Report of an epidemiological study. $J$ Periodontol 40: 5, 1969.

73. Svanberg, G., and Lindhe, J.: Vascular reactions in the periodontal ligament incident to trauma from occlusion. $J$ Clin Periodont 1: 58,1974

74. Svanberg, G.: Influence of trauma from occlusion on the periodontium of dogs with normal or inflamed gingiva. Ondontol Revy 25: $165,1974$. 
75. Svanberg, G., and Lindhe, J.: Experimental tooth hypermobility in the dog. Odontol Revy 24: 269, 1973.

76. Trott, J. R., and Love, B.: An analysis of localized gingival recession in 766 Winnipeg high school students. Dent Pract 16: 209, 1966.

77. Vale, J. D. F., and Ash, M. M.: Occlusal stability following occlusal adjustment. J Prosthet Dent 27: 515, 1972.

78. Waerhaug, J., and Hansen, E.: Periodontal changes incident to prolonged occlusal overload in monkeys. Acta Odontol Scand 24: 91, 1966.
79. Wentz, F. M., Jarabak, K. J., and Orban, B.: Experimental occlusal trauma imitating cuspal interferences. $J$ Periodontol 29: 117, 1958.

80. Woefter, C.: The prevalence and etiology of gingival recession. Periodont Abstracts 17: 45, 1969.

Send reprint requests to: Dr. Sigurd P. Ramfjord, Professor of Dentistry, University of Michigan, Ann Arbor, MI 48109.

\section{Abstracts}

\section{Adenoid Cystic Carcinoma of the Nose, Paranasal Sinuses, and PaLATE}

Miller, R. H., and Calcaterra, T. C.

Arch. Otolarynol. 106: 424, July, 1980.

In a study of 18 cases of adenoid cystic carcinoma in patients ages 13 to 78 years, seven cases involved the maxillary antrum, seven originated on the palate, and four were primary tumors of the nasal cavities. Initial symptoms were a mass or lump, pain, or nasal obstruction. Decreased visual activity was found in patients with sinus lesions. Only one patient had cervical node metastasis and radiographic evidence of pulmonary involvement. Surgery as the sole means of therapy was performed on nine patients and radiation therapy alone was utilized as the initial mode of treatment in two patients with antral tumors. Combined therapy was used for seven patients. Follow up was extended up to 10 years, and prognosis was shown to be poor. The poorest prognosis was for tumors of the minor salivary glands. Department of Otorhinolaryngology and Communicative Sciences, 1200 Moursund, Houston, TX. $77030 . \quad$ Dr. John Vrotsos

Ultrastructure of the Conjunctiva, Skin, and Gingrva

Messer, G., Harel, S., Erlich, B., Navon, R., Nemet, P., Sarnat, H., Shomrat, R., and Legum, C.

Arch. Pathol. Lab. Med. 104: 123, March, 1980.

A case of Sandhoff's disease with clinical symptoms typical of gangliosidosis was described in a child with multiple developmental disabilities including blindness and psychomotor retardation. Biochemical analysis included biopsy of the gingival papillae which were red and hypertrophic. Both hexosaminidase A and hexosaminidase B activities were deficient in the leukocytes and serum. Ultrastructural analysis of the conjunctiva, skin and gingiva as an aid for the diagnosis of Sandhoff's disease was recommended along with biochemical examination. Sandhoff's disease is a generalized lysosomal storage disease. Laboratory of Electron Microscopy, Municipal Governmental Medical Center, 6 Weizman Street, Tel Aviv, Israel 7065. Dr. John Vrotsos

A Multiprofessional Study of Patients With Myofascial PaIN-Dysfunction Syndrome. II

Helöe, B., and Heiberg, A. N. Acta Odontol. Scand. 38: 119, Number 2, 1980.

To improve diagnostic procedures and treatment planning for myofascial pain dysfunction syndrome, 108 patients were tested using a multiprofessional basis which included dental, psychiatric and physiologic characteristics. Questions asked the patients were history of oral symptoms, oral habits, their personal opinion of cause, what they expected of treatment, daily stress, reactions to nondental examiners. All the answers were related to psychiatric categorization, which was the capacity for interpersonal contact (CIC). Patients with severe CIC reported strong pain and an unstable occlusion. Patients with good CIC reported restriction of mandibular mobility and moderate pain on function. All patients reported fear of dislocation of the jaw. Most of the palpative findings by the dentist showed reports of pain with no differences found in oral habits. One third of the patients reported being in stress while being examined by the dentist and a great proportion of the patients reported positively to nondental physiotherapy. As a result of the study it was concluded that patients with complex MPD syndrome should be treated by specialists or on a multiprofessional basis by the dentist and psychiatrist. Bite (occlusal) instability and fear of jaw dislocation should be checked for the MPD syndrome. Institute of Community Dentistry, Dental Faculty, Blindern, Oslo 3, Norway.

Dr. Rafael Diaz-Mendez

The Histopathologic Features Which Follow Repeated applications of Tobacco Tar to Rat Lip Múcosa

Bastiaan, R. J. and Reade, P. C. Oral Surg., 49: 435, May, 1980.

To study and evaluate the histologic changes in rat lip mucosa exposed to repeated tobacco tar applications, 119 Sprague-Dawley rats were used. Two forms of tobacco tar were used, Tar A and Tar B, which had basic differences in concentration. Heat was also used in two forms; dry heat and a thermostatically controlled water bath. The rats were divided into six groups, three experimental and three control groups. After a designated period the rats were killed and histologic sections were made of lungs and lip mucosa. Nodules were examined in the lung tissue, and the lip mucosa was studied for keratinization, granular cell layer thickening, acanthosis, epithelial atypia, and inflammatory cells. The results showed that nodules appearing in the lungs were inflammatory in nature. The histologic sections showed a general irritational hyperplasia in oral mucosa from the tar with additional irritation caused by the heat. No capacity to initiate neoplastic activity was found. Department of Dental Medicine and Surgery, University of Melbourne, 711 Elizabeth Street, Melbourne, Victoria, 3000, Australia.
Dr. Rafael Diaz-Mendez 